\title{
Data communication for drone-enabled internet of things
}

\author{
Yousra Abdul Alsahib S. Aldeen, Haider Mohammed Abdulhadi \\ Department of Computer Science, College of Science for Women, University of Baghdad, Baghdad, Iraq
}

\begin{tabular}{|c|c|}
\hline Article Info & ABSTRACT \\
\hline Article history: & \multirow{10}{*}{$\begin{array}{l}\text { Internet of things (IoT) is one of the prominent emerged technology of } \\
\text { interconnected devices for people convenient and smart services. Recent } \\
\text { advancement in this area caused various new challenges especially } \\
\text { deployment of infrastructure. In order to fulfill the network requirements, the } \\
\text { dynamic and dedicated drone networks have designed as a cost effective and } \\
\text { flexible solution. The technologies of IoT and drone are emerged to collect, } \\
\text { forward the data for further process. Data communication among drones and } \\
\text { IoT infrastructure is new area of research where various different existing } \\
\text { protocol are used. However, still this area need attention due to mobility of } \\
\text { drones, obstacles and interferences in these networks. This paper proposes a } \\
\text { drone enabled data communication for internet of things (DDC-IoT) as a data } \\
\text { communication solution for IoT networks, data collection centers and drones. } \\
\text { The proposed data commination solution is tested in simulation to analyze its } \\
\text { performance especially for real time critical applications in terms of data } \\
\text { throughput and data delay. }\end{array}$} \\
\hline Received Dec 11, 2020 & \\
\hline Revised Mar 29, 2021 & \\
\hline Accepted Apr 14, 2021 & \\
\hline Keywords: & \\
\hline Communication & \\
\hline Data & \\
\hline Drone & \\
\hline IoT & \\
\hline Technology & \\
\hline
\end{tabular}

This is an open access article under the CC BY-SA license.

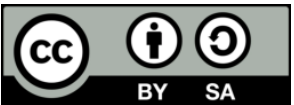

\section{Corresponding Author:}

Yousra Abdul Alsahib S. Aldeen

Department of Computer Science, College of Science for Women

University of Baghdad, Baghdad, Iraq

Email: yousraalkaalesi@gmail.com

\section{INTRODUCTION}

With rapid development of new technologies, smart intelligent systems and integrated networks open the new areas of research for better and cost effective services. There are various examples of new areas including internet of things (IoT), smart homes, smart transportation systems, smart grids, and augmented reality and drone technologies [1], [2]. The existing networks have suffered with various data communication issues due to congested areas, urban cities and infrastructure deployment. One of the example is IoT where the number of devices are connected with each other and able to communication and facilitate the users. This network provides more convenient services to users such as in smart home connected appliances, smart healthcare systems for patient take care, smart grid systems to manage the electricity, smart security things to secure the home form any type of security threat [3]. Due to this development of various technologies, the amount of data is generated by different technologies and a big part of this data belongs to IoT [1], [4], [5]. The concept of IoT comes from everyday objects, from fashionable devices to Industrial devices. Billions of devices all around the world communicated and sharing data through internet. In comping years' statistics show that more business and investment come in IoT sector. IoT have huge impact on over daily life drastically it shapes everything. It is predicted that till 2025, the impact of IoT reach to 6 trillion dollars.

In the context of data handling in IoT, the existing infrastructure based solution are suffered with cost, maintenance issues. Drone technology or unmanned aerial vehicle (UAV) is one of the advance and smart solution for data collection in IoT networks for in time data processing [6], [7]. Drone are autonomous robots used for data gathering, transportation, agriculture spray, safety and security, disaster management, and forest and weather management. Drones are also gained popularity in intelligent transportation system 
where these drone capture the accident scene from all angles before physical presence of people within less time [8], [9]. Drone are also utilized for fire-fighting purposes, package delivery, medical kit delivery on difficult sites where the human presence need more time [10], [11]. Figure 1 shows the drone enabled IoT networks and data communication process.

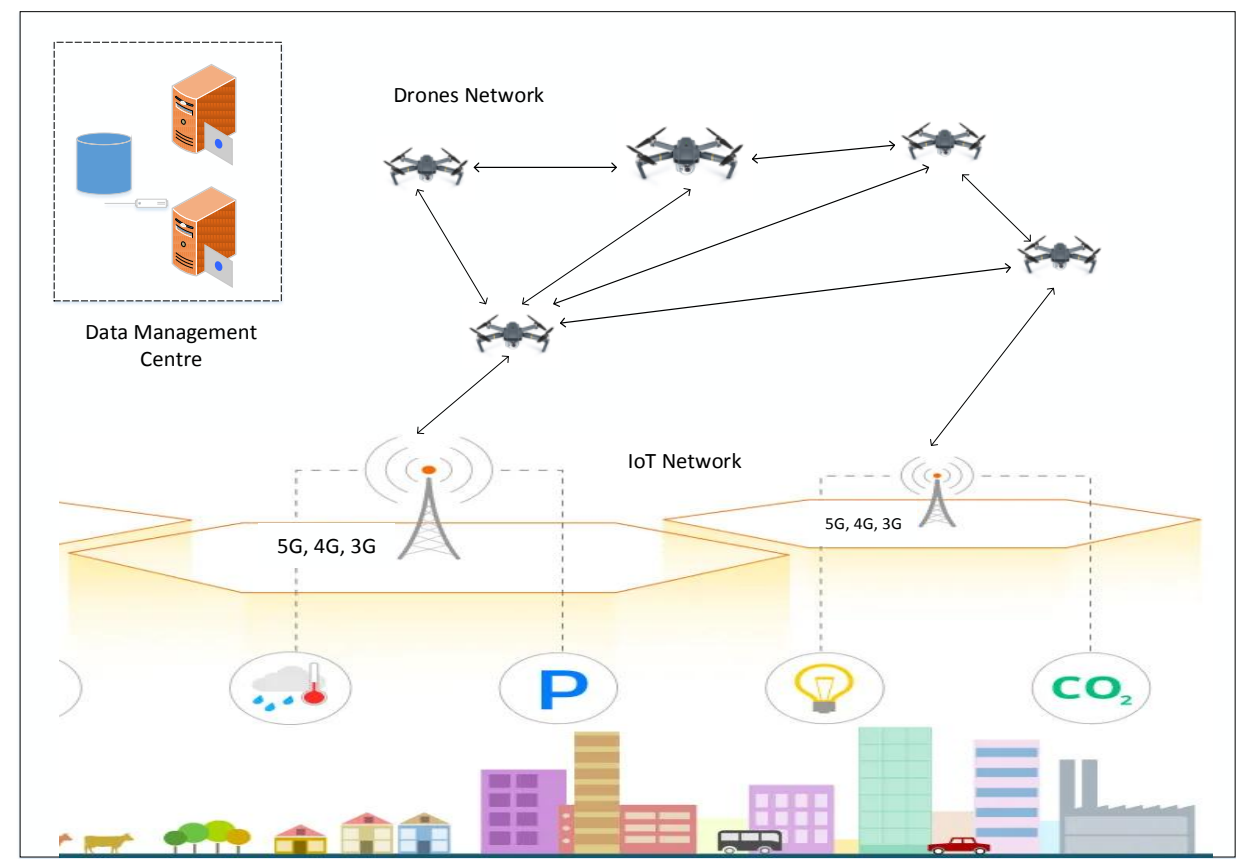

Figure 1. Drone enabled data communication with IoT

There are various notable benefits have observed in drone technologies such as control the pollution, energy saving, less risk, quick response and access time in emergencies, less cost solution, easy to handle and maintain. With many benefits the drone technologies areas and communication protocols have suffered with degradation issues, scalability and implementation issues, coverage areas issues, limited energy resources and physical security issues [12], [13]. This paper main focus is on data communication protocols between IoT and drone technologies. Drones are dynamically collect the IoT devices data and process it on real-time and send to data management centers [14], [15]. In this context, this paper presents the data communication solution called drone enabled data communication for internet of things (DDC-IoT) for drone enabled IoT networks. The other objectives of this paper are as follows:

- Discuss the existing solutions and their limitations

- Proposed a drone enable IoT data communication solution

- Analyzed the proposed work with state of the art solutions

The rest of the paper is organized as follows: Section 2 provides a related work in the area of drones in IoT networks. Section 3 presents the proposed data communication solution for IoT networks. The results and discussion present in Section4. The last section concludes the paper with future direction.

\section{RELATED WORK}

Many studies and solution have been designed to facilitate the IoT networks and overcome the data communication load by using smart sensor devices, infrastructure based solutions. However, these existing solutions are not able to overcome the IoT existing challenges. In this section, we discuss some of the existing solutions for IoT networks to analyze their limitations and challenges.

Mohamed, et al. [16] discussed the technical issues of drone enable technologies in smart cities environment. Authors discussed the difficulties of drone implementation in smart cities such as safety, privacy, and other legal and ethical issues. This new and attractive technology has various technical issues related to its hardware, software, data communication and other operations. Safety is one of the main concerns of these unmanned aerial vehicle (drone) because its presence in the air and among the buildings and close to people. The successful operations of this technology need more safety requirements. Security is 
another factor due to the organizations, industries and people confidential data. Drone need communication links with existing system for data communication and need specific requirements in terms of bandwidth, transfer rate, jitter and delay. Different types of data communication platform are used to facilitate the drones with ground networks such as satellite communication, cellular communication, and mesh networks. However, still these networks are suffered with these data communication solutions.

N. Hossein Motlagh, et al. [17], discussed the drone architecture for IoT networks operations. Authors also discussed about these drone technologies challenges related to physical collision, breaking the people privacy, and used or illegal purposes like drug smuggling. The data communication technologies in these networks are based on WiMAX, Wi-Fi, 3 to $5 \mathrm{G}$ standards. The drone is used these technologies to communicate between drones or with network devices. This paper presented a communication architecture and considered machine-to-machine data communication. However, this paper discussed the current scenario of drone technologies and its challenges and issues. This study does not discuss the proposer mechanism for drone enable technologies with other communication technologies.

Motlagh, et al. [18] presented a drone enable system for IoT network based on new emerging technologies. The proposed solution provides offloading video data processing process among drones and IoT devices. The proposed system is used for disaster relief management, capturing the images for site assessment and real time data communication. UAVs are integrating with IoT networks and provide a highlevel view. In addition, the authors also presented the UAV based crowd surveillance by using facial recognition tools. The proposed model is tested in real time network. The testbed results indicated the proposed model quick detection and recognition of suspicious people. However, these type of solutions are used for sensing or recognizing some objects but difficult for precisely data handling.

Authors in [19], proposed a queuing delay and transmission delay (QDTD) routing protocol. This protocol is based on queuing delay and transmission delay strategies for reliable data communication. Authors claimed that the proposed solution has better results to reduce the data delay and increase the data throughput in the networks. This solution is especially designed for drones enable IoT networks. This solution is feasible to handle the load to alleviate the network congestion, transmission delay and queuing delay. However, this solution has complex processing method and takes more computation time which leads to data delay and decreasing the data throughput.

Mozaffari, et al. [20] presented an optimal trajectory and deployment of multiple UAVs in IoT networks for data collection. The proposed solution provides reliable data communication with less energy consumption. This study uses swarm drones to minimizes the transmission power from IoT devices. Each drone is used to deliver the services to the cluster of IoT devices on the ground. The proposed solution is a novel framework based on clustering method for optimal deployment of UAVs and obtained the trajectories used in UAVs to serve the mobile IoT devices. The simulation results indicated that the proposed solution decreased transmit power and network will operational for long time. However, this type of solution is useful for static networks where the clustering is easy to deploy.

A. K. Saha, et al [21], presented a UAVs enable solution for IoT network by using digital cameras, sensors, and data communication devices. This system provides integrating solutions to achieve the easy, efficient, precise and monitoring. The proposed solution is based on machine learning concepts to increase the scope of further improvement. This system is designed for agriculture crop monitoring with less energy consumption. With the help of localization mapping, the proposed system captures high resolution images and for crop management. The drone provides more precise and accurate ground situation and adjust and measure the distance from terrain and calculate the depth level of water, stress level of crop. However, this type of system is limited in scope and high resolution images capturing need more bandwidth and strong infrastructure on the ground for drone communication. Table 1 shows the comparison of discussed studies.

The various surveys have been conducted to discuss the drone's benefits and compatibility with IoT but still this area is in blur shape where the most significant area is data communication among drones and IoT networks. In Table 1, most of the existing solutions are not offering security and privacy factors. Some of them offer energy management and consumption parameters.

Table 1. Comparison of discussed studies

\begin{tabular}{cclccc}
\hline S\# & Solution & \multicolumn{1}{c}{ Field } & Energy Consumption & Data Collection & Security and Privacy \\
\hline 1 & {$[16] / 2018$} & Drone enable Smart Cities & No & Yes & No \\
2 & {$[17] / 2016$} & Drone enable IoT & No & No & No \\
3 & {$[18] / 2017$} & Drone enable IoT & No & Yes & No \\
4 & {$[19] / 2017$} & Drone Routing & No & Yes & No \\
5 & {$[20] / 2016$} & Drone enable IoT & Yes & Yes & No \\
6 & {$[21] / 2018$} & Drone enable IoT Agriculture & Yes & & \\
\hline
\end{tabular}

Indonesian J Elec Eng \& Comp Sci, Vol. 22, No. 2, May 2021 : 1216 - 1222 


\section{PROPOSED SOLUTION}

This section presents the detail of proposed solution called drone enabled data communication for internet of things (DDC-IoT). The proposed solution provides an efficient data communication for IoT networks such as for agriculture fields, disaster relief, and smart building. The traditional system is based on deployed sensors nodes which are costly and complex system. DDC-IoT provides cost effective, reliable and maintenance free solution for data collection. In this system the drones are behaving like access points and flies over the filed area and connect with base station and sensor devices at the IoT network side. The DDCIoT design is based on communication IEEE 802.11 standard and low power IoT technologies including IEEE 802.15.4 and SigFox and Lora. The proposed model is based on two types of data communication drone-to-drone and drone to IoT networks for data gathering. These two proposed data communication systems are explaining in next subsections.

\subsection{Drone-to-Drone Data Communication}

In proposed DDC-IoT protocol, we use two parameters transmission power (TP) and beacon frequency $(\mathrm{BF})$. Beacon frequency should be at level because high frequency leads to interference and low frequency leads to packet dropping issues. We are using the beacon frequency as per network situation by using channel situation (CS), and queue factor (QF). The proposed DDC-IoT is divided into two main phases called Initialization phase and process phase. The phases are discussing in next sub sections.

\subsubsection{Initialization Phase}

In this phase, the local information including the update of one hop neighbors and check the communication channel estimation. The drones gather the neighbor drone's information and create an information list $\mathrm{D}(\mathrm{OHN})$. This phase estimates the queue status and communication channel usage. The maximum channel capacity measures by MCcapacity. This capacity shows the maximum beacons in queue without channel overload. Threshold ratio is used to evaluate the channel capacity. Equation 1 shows the effective channel capacity.

$$
\alpha=\beta \cdot M C_{\text {capacity }}
$$

Where $\alpha$ denotes the channel capacity, $\beta$ denotes threshold ratio and $M C_{\text {capacity }}$ denoted the maximum channel capacity. The neighbor drones help to compute the QF and estimates the ques status and beacon frequency of drones and its neighbor drones as shows in (2).

$$
Q F=B F_{i}+\left(\sum_{j}^{\mathrm{OHN}(\mathrm{i})} R B_{j}\right)
$$

Where the QF denotes the queue factor, BF denotes the beacon frequency, the (2) are used to evaluate the channel congestion situation. When the $\mathrm{QF} \leq \alpha$, the congestion free situation is defined.

\subsubsection{Process Phase}

In this phase, the all the defined and important parameters are evaluated to check the network condition and other data transmission parameters. This phase also adjusts the beacon frequency as per dynamic nature of the drone nodes. The beacon frequency is adjusted with the help of (3).

$$
D F_{i} \leftarrow D F_{i}-D F_{i} * \Delta A F
$$

Where the $D F_{i}$ denotes the drone frequency for each drone $i$, and $\triangle A F$ denotes the adopted fraction. After this, the next step is set transmission power based on adaptation fraction and Drone Transmission (DT). Figure 2 shows the Algorithm 1 for simple drone-to-drone data communication process and adjust transmission power and beacon frequency to avoid any sort of channel congestion.

\begin{tabular}{lll}
\hline & & \multicolumn{1}{c}{ Algorithm 1} \\
\hline 1 & Input & One hop searching \\
2 & Output & Update beacon transmission power \\
3 & do & Drone computes $Q F$ \\
4 & do & Evaluate $C S$ \\
5 & do & Drones update beacon drone frequency $(D F)$ based on: \\
6 & do & DF $\leftarrow$ DF $-D F_{i} * \Delta A F$ \\
7 & & Drones adjust beacon drone transmission $(D T)$ \\
8 & end procedure & \\
\hline
\end{tabular}




\subsection{Drone to IoT Network Data Communication}

In this section, the drone-to-drone data communication is further connected to IoT networks by suing $5 \mathrm{G}$ based network infrastructure. The $5 \mathrm{G}$ based services and technologies are able to provide a fully connected, fast and effective platform to make the data communication possible between drones and IoT smart devices. This level provides network resources and scheduling methods for a better network environment [22]. This level also offers real-time, on-demand, reconfigurable, end-to-end coordination, and intelligent services. High frequency based on band $6 \mathrm{GHz}$ with a $10 \mathrm{~Gb} / \mathrm{s}$ is used for data rate. Another feature of this technology is providing an efficient IoT devices data communication infrastructure without any extra and costly hardware-specific methods. This phase also useful for low latency around $1 \mathrm{~ms}$ and handling 1 million devices $/ \mathrm{km} 2$. The deployment of new services is set at less time (around 1 hour).

\section{RESULTS AND DISCUSSION}

In this section, we discuss the simulation setup require to evaluate the proposed DDC-IoT solution with QDTD [19] state of the art solutions. Number of simulations run help to evaluate the proposed solution in terms of data delivery, end-to-end delay and network overhead. We selected Ns-3 simulation which is a network simulator based on open source discrete event network features. This simulation is useful for data transmission protocols and implementation of TCP/IP standard. NS-3 simulator provides a C++ and python scripting, alignment with real time system [23-25]. Table 2 shows the simulation parameters adopted to test the proposed solution.

Table 2. Simulation parameters

\begin{tabular}{llc}
\hline S\# & \multicolumn{1}{c}{ Parameters } & Values \\
\hline 1 & Network Size & $100 \times 100$ \\
2 & No of Drones & 12 \\
3 & No of IoT Devices & 20 \\
4 & Communication Standard & $5 \mathrm{G}$ \\
5 & Propagation Model & Two-Ray \\
6 & No of Simulation Runs & 10 \\
7 & Transmission Power & $12 \mathrm{dBm}$ \\
8 & Radio Interface & IEEE 802.11p \\
\hline
\end{tabular}

\subsection{Data Throughput}

In these experiments, we evaluate the data throughput to analyze the performance of proposed DDC-IoT solution with QDTD. For these experiments, we used to analyze the effects of communication channel busyness ratio and evaluate the data throughput. We selected different random seeds and conduct experiments. We tracked the data packets and measure the data throughput in Mbits/s by using different values of channel busyness ratio. Channel busyness value defined as a fraction of constant time unit where the channel in no idle situation. Figure 2 clearly shows the better performance of proposed DDC-IoT solution compared to QDTD even with more channel busyness situation. When the channel busyness ratio reached to 0.5 then the proposed solution is showing better results compared to existing solution.

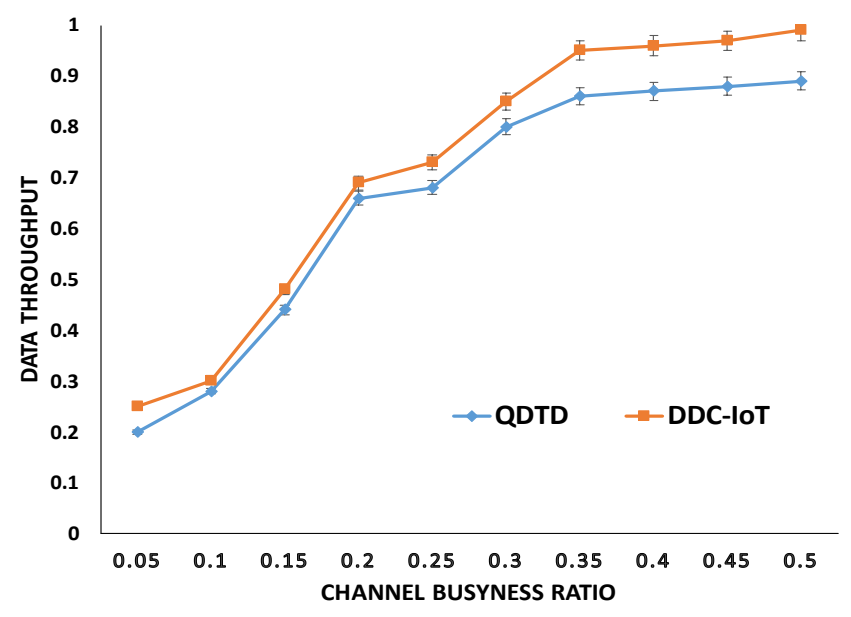

Figure 2. Data Throughput Analysis 


\subsection{Data Delay}

These experiments show the data delay impact in networks when the drones are communicating with IoT networks to gather the data and responds to base station. In these experiments, we evaluate the data delay to analyze the performance of proposed DDC-IoT solution with QDTD. For these experiments, we analyzed the channel busyness ratio to check the effects of communication channel busyness ratio and evaluate the data delay. We selected different random seeds and conduct experiments. We tracked the data packets and measure the data throughput in Mbits/s by using different values of channel busyness ratio. Channel busyness value defined as a fraction of constant time unit where the channel in no idle situation. Figure 3 clearly shows the better performance of proposed DDC-IoT solution compared to QDTD even with more channel busyness situation. The proposed solution DDC-IoT performance is better even with more channel busyness ratio. When the channel busyness ratio reached to 0.5 then the proposed solution is showing better results compared to existing solution and has less delay in the network. Both the results are showing positive performance in terms of data delay and data throughput.

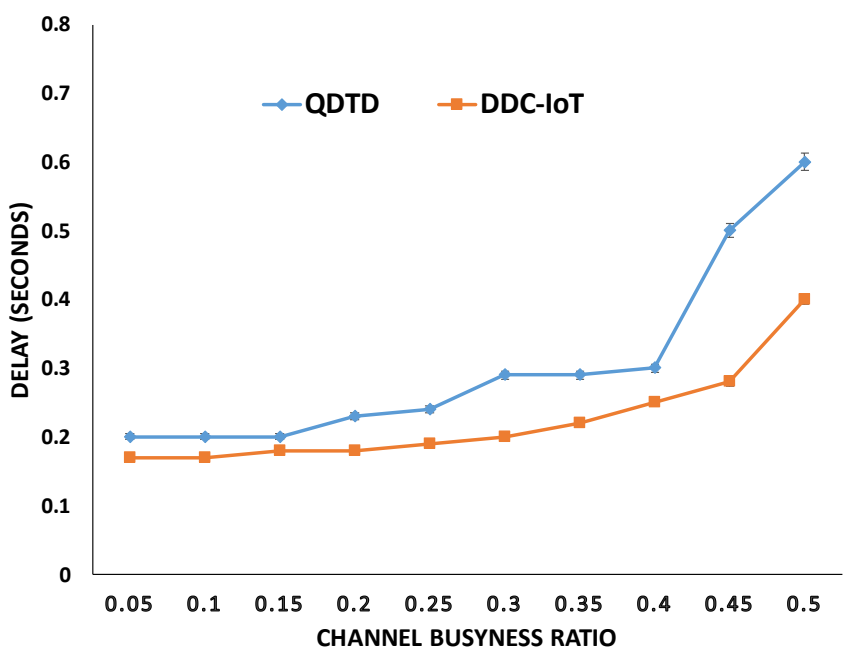

Figure 3. Data Delay Analysis

\section{CONCLUSION}

Internet of things (IoT) is a connected devices network where a number of devices generated the data. Data gathering is one of the challenging tasks in these networks. Drones are one of the cost effective and manageable solution for data gathering from IoT devices and send for further analysis by using fast and reliable data communication standards. There are various challenges have been faced IoT networks such as costly infrastructure deployment, data delay, unreliable data communication. In order to address these challenges, the dynamic and dedicated drone networks have designed as a cost effective and flexible solution. Data communication among drones and IoT infrastructure is another challenge for existing network protocols. We proposed a drone enabled data communication for internet of things (DDC-IoT) solution for data communication among drones and IoT devices. The proposed solution is controlling the beacon frequency and transmission power and improve data deliver in less time and enhance data throughput. The proposed solution is evaluated in simulation where the results indicated the better performance of proposed solution as compared to existing protocols. In future, we test the proposed protocol with more complex networks and use more performance parameters.

\section{REFERENCES}

[1] K. N. Qureshi, M. U. Bashir, J. Lloret, and A. Leon, "Optimized Cluster-Based Dynamic Energy-Aware Routing Protocol for Wireless Sensor Networks in Agriculture Precision," Journal of Sensors, 2020, doi: 10.1155/2020/9040395.

[2] K. N. Qureshi, S. Din, G. Jeon, and F. Piccialli, "Link Quality and Energy Utilization Based Preferable Next Hop Selection Routing for Wireless Body Area Networks," Computer Communications, vol. 149, pp. 382-392, 2020, doi: 10.1016/j.comcom.2019.10.030.

[3] K. N. Qureshi, G. Jeon, and F. Piccialli, "Anomaly Detection and Trust Authority in Artificial Intelligence and Cloud Computing," Computer Networks, vol. 184, 2020, doi: 10.1016/j.comnet.2020.107647. 
[4] K. N. Qureshi, F. Bashir and A. H. Abdullah, "An Energy and Link Aware Next Node Selection Protocol for Body Area Networks," 2018 International Conference on Information Networking (ICOIN), 2018, pp. 721-726, doi: 10.1109/ICOIN.2018.8343213.

[5] M. S. Aliero, K. N. Qureshi, M. F. Pasha, I. Ghani, and R. A. Yauri, "Systematic Mapping Study on Energy Optimization Solutions in Smart Building Structure: Opportunities and Challenges," Wireless Personal Communications, pp. 1-37, 2021, doi: 10.1007/s11277-021-08316-3.

[6] F. Mohammed, A. Idries, N. Mohamed, J. Al-Jaroodi and I. Jawhar, "UAVs for Smart Cities: Opportunities and Challenges," 2014 International Conference on Unmanned Aircraft Systems (ICUAS), 2014, pp. 267-273, doi: 10.1109/ICUAS.2014.6842265.

[7] J.-M. Jo, "An Efficient MANET Routing Protocol for the Drone Delivery Communication Network System," The Journal of the Korea institute of electronic communication sciences, vol. 10, no. 9, pp. 973-978, 2015, doi: 10.13067/JKIECS.2015.10.9.973.

[8] K. N. Qureshi, A. Ahmad, F. Piccialli, G. Casolla, and G. Jeon, "Nature-Inspired Algorithm-Based Secure Data Dissemination Framework for Smart City Networks," Neural Computing and Applications, pp. 1-20, 2020, doi: 10.1007/s00521-020-04900-z.

[9] K. A. M. Abid Ali, A. Alabaichi, and A. S. Abbas, "Dual Method Cryptography Image by Two Force Secure and Steganography Secret Message in IoT," Telkomnika (Telecommunication, Computing, Electronics and Control), vol. 18, no. 6, pp. 2928-2938, 2020, doi: 10.12928/TELKOMNIKA.v18i6.15847.

[10] Rathy G. A., Sivasankar P., and T. Z. Fadhil, "An efficient IoT based biomedical health monitoring and Diagnosing System Using myRIO," Telkomnika (Telecommunication, Computing, Electronics and Control), vol. 18, no. 6, pp. 3050-3057, 2020, doi: 10.12928/telkomnika.v18i6.14375.

[11] A. M. Al-Ghaili, H. Kasim, M. Othman, and W. Hashim, "QR Code Based Authentication Method for IoT Applications Using Three Security Layers," Telkomnika (Telecommunication, Computing, Electronics and Control), vol. 18, no. 4, pp. 2004-2011, 2020, doi: 10.12928/telkomnika.v18i4.14748.

[12] S. H. Alsamhi, O. Ma, and M. S. Ansari, "Predictive Estimation of the Optimal Signal Strength from Unmanned Aerial Vehicle Over Internet of Things Using ANN," arXiv preprint arXiv:1805.07614, 2018.

[13] C. Bunse and S. Plotz, "Security Analysis of Drone Communication Protocols," in International Symposium on Engineering Secure Software and Systems, 2018, pp. 96-107, doi: 10.1007/978-3-319-94496-8_7.

[14] M. Mozaffari, W. Saad, M. Bennis and M. Debbah, "Mobile Unmanned Aerial Vehicles (UAVs) for EnergyEfficient Internet of Things Communications," in IEEE Transactions on Wireless Communications, vol. 16, no. 11, pp. 7574-7589, Nov. 2017, doi: 10.1109/TWC.2017.2751045.

[15] T. Lagkas, V. Argyriou, S. Bibi, and P. Sarigiannidis, "UAV IoT Framework Views and Challenges: Towards Protecting Drones as "Things"," Sensors, vol. 18, no. 11, 2018, doi: 10.3390/s18114015.

[16] N. Mohamed, J. Al-Jaroodi, I. Jawhar, A. Idries, and F. Mohammed, "Unmanned Aerial Vehicles Applications in Future Smart Cities," Technological Forecasting and Social Change, vol. 153, 2020, doi: 10.1016/j.techfore.2018.05.004.

[17] N. Hossein Motlagh, T. Taleb and O. Arouk, "Low-Altitude Unmanned Aerial Vehicles-Based Internet of Things Services: Comprehensive Survey And Future Perspectives," in IEEE Internet of Things Journal, vol. 3, no. 6, pp. 899-922, Dec. 2016, doi: 10.1109/JIOT.2016.2612119.

[18] N. H. Motlagh, M. Bagaa and T. Taleb, "UAV-Based IoT Platform: A Crowd Surveillance Use Case," in IEEE Communications Magazine, vol. 55, no. 2, pp. 128-134, February 2017, doi: 10.1109/MCOM.2017.1600587CM.

[19] P. Yang, X. Cao, C. Yin, Z. Xiao, X. Xi and D. Wu, "Routing protocol design for Drone-Cell Communication Networks,"2017 IEEE International Conference on Communications (ICC), 2017, pp. 1-6, doi: 10.1109/ICC.2017.7996484.

[20] M. Mozaffari, W. Saad, M. Bennis and M. Debbah, "Mobile Internet of Things: Can UAVs Provide an EnergyEfficient Mobile Architecture?," 2016 IEEE Global Communications Conference (GLOBECOM), 2016, pp. 1-6, doi: 10.1109/GLOCOM.2016.7841993.

[21] A. K. Saha, et al., "IoT-based Drone for Improvement of Crop Quality in Agricultural Field," 2018 IEEE 8th Annual Computing and Communication Workshop and Conference (CCWC), 2018, pp. 612-615, doi: 10.1109/CCWC.2018.8301662.

[22] G. A. Akpakwu, B. J. Silva, G. P. Hancke and A. M. Abu-Mahfouz, "A Survey on 5G Networks for the Internet of Things: Communication Technologies and Challenges," in IEEE Access, vol. 6, pp. 3619-3647, 2018, doi: 10.1109/ACCESS.2017.2779844.

[23] J. Lee, K. Kim, H. Kim and H. Kim, "Devising Geographic Diffusion for Drone Networks," 2016 Eighth International Conference on Ubiquitous and Future Networks (ICUFN), 2016, pp. 76-78, doi: 10.1109/ICUFN.2016.7536985.

[24] G. F. Riley and T. R. Henderson, "The ns-3 network simulator," in Modeling and tools for network simulation, Berlin, Heidelberg, Germany: Springer, 2010, pp. 15-34, doi: 10.1007/978-3-642-12331-3_2.

[25] G. J. A. M. Carneiro, "NS-3: Network simulator 3," in UTM Lab Meeting April, vol. 20, pp. 4-5, 2010. 